DOI: http://dx.doi.org/10.1590/1981-7746-sol00180

\title{
O PROCESSO DE TRABALHO DO NÚCLEO AMPLIADO DE SAÚDE DA FAMÍLIA E ATENÇÃO BÁSICA
}

\author{
WORK PROCESS OF THE FAMILY HEALTH AND PRIMARY \\ HEALTH CARE EXPANDED SUPPORT CENTER
}

\begin{abstract}
Isabelle Cristina Borba da Silva (iD) (https://orcid.org/0000-0001-7148-4266) ${ }^{1}$, Laís Alves Bernadino da Silva (https://orcid.org/0000-0003-2412-2072) ${ }^{2}$, Ana Maria Gondim Valença (iD) (https://orcid.org/0000-0001-8460-3981) ${ }^{3}$, Juliana Sampaio (iD) (https://orcid.org/0000-0003-0439-5057) ${ }^{1}$
\end{abstract}
${ }^{1}$ Universidade Federal da Paraíba, Centro de Ciências Médicas, João Pessoa, Paraíba, Brasil. <isabelleborba8@gmail.com>

\footnotetext{
${ }^{2}$ Universidade Federal da Paraíba, Departamento de Clínica e Odontologia Social, João Pessoa, Paraíba, Brasil.

${ }^{3}$ Universidade Federal da Paraíba, Departamento de Promoção da Saúde, João Pessoa, Paraíba, Brasil.
}

\begin{abstract}
Resumo O crescimento da implantação do Núcleo Ampliado de Saúde da Família e Atenção Básica na Paraíba e sua influência na melhoria da qualidade da assistência das equipes de atenção primária torna relevante conhecer a organização do processo de trabalho destes núcleos. O estudo analisou ações de planejamento, oferta de formação inicial e de educação permanente e integração com as equipes apoiadas. Metodologicamente é descritivo, quantitativo, de recorte transversal. Os dados foram obtidos do módulo IV do $2^{\circ}$ ciclo de avaliação externa do Programa de Melhoria e Acesso da Qualidade da Atenção Básica. O mapa coroplético foi construído em TabWin e a análise descritiva realizada no IBM SPSS Statistics 20.0. Quanto à oferta de formação inicial e educação permanente para o Núcleo, notou-se que as equipes que receberam a primeira $(50 \%)$ tiveram maior frequência de oferta da segunda (74,1\%), e estavam localizadas em municípios de maior porte. A formação inicial teve como atividades mais frequentes as oficinas de capacitação/reunião informativa $(20,7 \%)$ e curso introdutório (20,7\%). Há uma fragilidade nos registros e na execução do planejamento sistematizado, principalmente no monitoramento e avaliação das ações. A integração entre as equipes Núcleo de Apoio à Saúde da Família e as equipes de referência é frágil e foge do pressuposto conceitual do apoio matricial.
\end{abstract}

Palavras-chave atenção básica; saúde da família; planejamento em saúde; educação permanente.
Abstract The increase in the implementation of the Family Health and Primary Health Care Expanded Support Center in the state of Paraíba, Brazil, and its influence on the improvement in the quality of the assistance of the primary health care teams makes it relevant to get to know the organization of the work process within these centers. The study analyzed the planning actions, the offer of initial training and ongoing education, and the integration with the supported teams. Methodologically, it is a descriptive, quantitative and cross-sectional study. The data was obtained from module IV of the 2nd cycle of external evaluation of the Program for the Improvement of the Access to and Quality of Primary Health Care (Programa de Melhoria do Acesso e da Qualidade da Atenção Básica, in Portuguese). The corochromatic map was developed using the TabWin software, and the descriptive analysis was made using the IBM SPSS Statistics software, version 20.0. Regarding the offer of initial training and ongoing education for the Center, we observed that the teams that underwent the former $(50 \%)$ were more likely to be offered the latter $(74.1 \%)$, and the courses were offered in larger municipalities. The initial training had as its most common activities the qualification/informational meeting workshops (20.7\%) and the introductory course $(20.7 \%)$. There is a weakness in the records and in the execution of the systematized planning, mainly regarding the monitoring and assessment of the actions. The integration between the Family Health Support Center teams and the reference teams is weak and not in line with the conceptual goals of the matrix support.

Keywords primary health care; family health; planning in health; ongoing education. 


\section{Introdução}

Apesar da existência de um aparato legal regulamentador das políticas públicas no Brasil, a Atenção Básica $(\mathrm{AB})$ à saúde ainda enfrenta diversos desafios para sua consolidação como principal estratégia assistencial. Destacam-se, entre elas, a garantia do acesso aos serviços e da resolutividade dos problemas de saúde da população.

A AB é caracterizada por um conjunto de ações de saúde voltadas ao individuo, família e coletividade, que envolve a promoção, proteção e recuperação da saúde. Ela busca desenvolver os princípios doutrinários de universalidade, integralidade e equidade da assistência, e deve ser porta principal de acesso aos usuários do sistema e ordenadora de redes de cuidado. Portanto, ela visa à continuidade do cuidado, constituição de vínculo, acessibilidade, responsabilização, humanização e participação social (Brasil, 2017b).

$\mathrm{A} A B$ expandiu-se no país por meio da dinâmica de prestação de cuidados primários centrada na família e comunidade, e tem se consolidado com a implantação da Política Nacional de Atenção Básica (PNAB). Preconiza-se que tenha capacidade de resolver pelo menos $80 \%$ dos problemas apresentados pelos usuários sob sua responsabilidade, por meio de ações sanitárias e práticas gerenciais democráticas (Martiniano et al., 2013; Campos et al., 2014).

$\mathrm{Na}$ perspectiva de fortalecimento da $\mathrm{AB}$, o apoio matricial (AM) é proposto como dispositivo para a reorganização do processo de trabalho em saúde, pela oferta de apoio técnico especializado aos profissionais das equipes de referência. Esta estratégia de gestão do trabalho busca fomentar a interlocução da equipe de saúde com a rede de serviços disponíveis, com ampliação da clínica e de sua capacidade resolutiva, com base na lógica dialógica e reflexiva dos processos de trabalho instituídos (Campos e Domitti, 2007).

Neste contexto, para potencializar a oferta de serviços disponíveis à população, possibilitar a integralidade no contexto da $\mathrm{AB}$ e compartilhar a responsabilidade da assistência à saúde, o Ministério da Saúde criou, em 2008, o Núcleo de Apoio à Saúde da Família (Nasf), atualmente denominado Núcleo Ampliado de Saúde da Família e Atenção Básica (Nasf-AB) (Brasil, 2017a). Seu propósito é apoiar as Equipes de Saúde da Família (EqSF) e de Atenção Básica (EqAB) com o objetivo de fortalecê-las em um trabalho integrado, com ações compartilhadas e interdisciplinares, a fim de aumentar a capacidade resolutiva de toda a equipe (Silva, I. et al., 2017).

Nesta perspectiva, o Nasf-AB consiste em uma estratégia criada para potencializar a resolutividade da $\mathrm{AB}$, com ações baseadas em diretrizes, tais como: interdisciplinaridade e intersetorialidade; desenvolvimento de noção territorialização; integralidade do cuidado, participação social, educação popular e promoção da saúde, configurando-se ainda como potente espaço de educação permanente (Brasil, 2010; França et al., 2017). 
Em contraposição aos modelos convencionais de prestação de cuidados, que optam pela assistência especializada, fragmentada e individual, a proposta do trabalho do Nasf-AB busca superar essa lógica baseando-se na construção de uma rede de atenção integral e resolutiva que articule os diferentes níveis assistenciais, sob coordenação das equipes de referência (Brasil, 2017b).

As equipes Nasf-AB (EqNASF-AB) agrupam profissionais de diferentes áreas do conhecimento, sob a forma de apoio matricial e com vistas à garantia da integralidade. Teórico-metodologicamente, a ação deste núcleo com as EqSF/ EqAB deve pautar-se nas seguintes ferramentas: Projeto Terapêutico Singular, Apoio Matricial e Clínica Ampliada. No estado da Paraíba, a composição das EqNasf-AB se dá principalmente por fisioterapeutas, nutricionistas, psicólogos, assistentes sociais e educadores físicos (Brasil, 2010, Silva, L. et al., 2017).

Assim como o Nasf-AB, o Programa Nacional de Melhoria do Acesso e da Qualidade da Atenção Básica (PMAQ-AB) consiste em uma estratégia para potencializar a AB. Sua pretensão é induzir a ampliação da capacidade gestora em ofertar serviços qualificados e acessíveis, conforme as reais necessidades da população in loco. Para tanto, visa permitir uma releitura e redirecionamento da $\mathrm{AB}$, com vistas à acessibilidade e resolutividade das práticas do cuidar (Brasil, 2013).

O PMAQ-AB é um programa nacional, fundamentado pela PNAB, associado à estratégia ministerial 'Saúde Mais Perto de Você'. Foi criado pela portaria n. 1.654 GM/MS, de 19 de julho de 2010, para estimular a ampliação do acesso e a qualificação da assistência à saúde e gestão desenvolvidas no âmbito da AB (Pinto, Sousa e Ferla, 2014). Propõe induzir mudanças e adequações, de maneira transparente e efetiva, nas ações assistenciais, gerenciais e governamentais direcionadas à $\mathrm{AB}$ no território nacional. O PMAQ tem contribuído para melhoria da qualidade dos serviços na $A B$, à medida que tem fortalecido aspectos como planejamento, avaliação e registro das ações em saúde, além da ampliação da valorização profissional por intermédio dos incentivos financeiros. (Chimara, Pain e Storopoli, 2013; Bertusso, 2017).

Dentre os indicadores da avaliação externa da $\mathrm{AB}$ pelo $\mathrm{PMAQ}$, visualizase no módulo IV do $2^{\circ}$ ciclo, informações sobre o processo de trabalho das EqNasf-AB que permitem responder a seguinte questão: Como se dá a organização do processo de trabalho das EqNasf-AB? O estado da Paraíba se destaca no cenário nacional por ter uma cobertura estimada de 94,64\% da população, com 1.354 EqSFs implantadas. Além disto, possui 273 EqNasf$\mathrm{AB}$ cadastradas, das quais 142 são do tipo I, 55 do tipo II e 76 do tipo III, a quarta posição nacional em números absolutos de EqNasf-AB (Dab, 2017).

Dada à importância do Nasf-AB como estratégia de fortalecimento da $\mathrm{AB}$ no estado da Paraíba, e a necessidade de compreensão de sua potencial influência na melhoria da qualidade da assistência, é relevante analisar a organização do processo de trabalho destas equipes, a fim de reconhecer 
suas fragilidades e potencialidades. O reconhecimento destas pelas equipes e gestores, principalmente as relacionadas às condições gerais de trabalho, às ações de planejamento e à integração com as equipes de referência, pode subsidiar a criação de estratégias de fortalecimento do apoio matricial e potencializar o aumento da resolutividade na AB. Nesta perspectiva, este estudo objetiva analisar a organização do processo de trabalho das EqNasf$\mathrm{AB}$, com ênfase nas ações de planejamento, na oferta de formação inicial e de educação permanente para as EqNasf-AB e na integração com as EqSF/ EqAB apoiadas.

\section{Metodologia}

Trata-se de um estudo descritivo, com abordagem quantitativa, de recorte transversal. Foi realizado com base nos dos dados do PMAQ-AB obtidos com as EqNasf-AB que fornecem $\mathrm{AM}$ às EqSF/EqAB no estado da Paraíba.

Na Paraíba, a avaliação externa do PMAQ-AB foi realizada sob coordenação da Universidade Federal da Paraíba, com o apoio da Universidade Federal de Campina Grande. A equipe de avaliação foi formada por seis professorescoordenadores de campo; oito supervisores de campo e 54 avaliadores de qualidade, além da coordenação geral e da equipe de apoio técnico.

Este estado é formado por 223 municípios divididos em quatro macrorregiões de saúde e 16 regiões de saúde. Deste total de municípios apenas um não participou do segundo ciclo de avaliação externa do PMAQ-AB.

A avaliação externa foi realizada durante os meses de fevereiro a maio de 2014. Foram avaliadas 116 EqNasf-AB, das 208 existentes na Paraíba em fevereiro de 2014 (Dab, 2014). As equipes respondentes se distribuíam em 76 municípios do estado, dos quais 4 eram de grande porte (João Pessoa, Campina Grande, Patos e Santa Rita), 13 de médio porte e 59 de pequeno porte. As equipes avaliadas responderam ao Módulo IV do $2^{\circ}$ ciclo de avaliação externa do PMAQ-AB e constituem a amostra do presente estudo.

$\mathrm{O}$ instrumento de coleta do $2^{\circ}$ ciclo de avaliação externa do PMAQ-AB, em seu módulo IV, aplicado aos profissionais das Nasf-AB do estado da Paraíba, contempla elementos (variáveis) que tornaram exequível a análise sobre o processo de trabalho destas equipes, relacionados à: oferta de educação permanente e formação inicial; planejamento das ações do Nasf-AB; organização da agenda; organização do apoio matricial às EqSF/EqAB; gestão da demanda e da atenção compartilhada e registro de atividades.

Os dados foram organizados em planilhas eletrônicas. Posteriormente, foi realizada a limpeza do banco de dados, a fim de eliminar falhas. Todas as variáveis com mais de $10 \%$ de perdas foram desconsideradas do banco. 
Empregou-se o software TabWin para construção do mapa coroplético. A análise descritiva (frequências absolutas e relativas) dos dados foi realizada com auxílio do software IBM SPSS Statistics 20.0.

\section{Resultados e discussão}

De acordo com a portaria ministerial n. 154 de 2008, há três modalidades possíveis de EqNasf-AB: EqNasf-AB I, vinculado a no mínimo cinco e no máximo nove EqSF/EqAB; EqNasf-AB II, vinculado a no mínimo três e no máximo quatro EqSF/EqAB e EqNasf-AB III, que pode vincular-se a um ou dois EqSF/EqAB, agregando-se a essas de modo a se tornarem equipes ampliadas (Brasil, 2012b).

Dentro da lógica de financiamento do Ministério da Saúde, cada município pode implantar apenas uma das modalidades de EqNasf-AB. Para as modalidades 2 e 3, apenas uma equipe por município é financiada. Dentro dessas instruções, cada município implanta as EqNasf-AB conforme o tamanho de sua população e número de EqSF/EqAB implantadas (Brasil, 2012b).

O estado da Paraíba se destaca em cenário nacional pelo quantitativo absoluto de EqNasf-AB implantadas em seus municípios. Das 116 equipes participantes do $2^{\circ}$ ciclo de avaliação externa do PMAQ-AB, $81,8 \%$ pertenciam à modalidade EqNasf-AB I, 17,3\% à II e 0,9\% à III.

A análise do módulo IV do $2^{\circ}$ ciclo de avaliação externa do PMAQ-AB possibilitou a problematização de aspectos inerentes ao processo de trabalho das EqNasf-AB, em especial os relacionados à oferta de formação inicial e de educação permanente para estas equipes, ao processo de planejamento, monitoramento e avaliação das ações e a integração das EqNasf-AB com as $\mathrm{EqSF} / \mathrm{EqAB}$ apoiadas.

\section{Oferta de formação inicial e de educação permanente para as EqNasf-AB}

A educação no trabalho em saúde se dá em duas modalidades: a educação permanente (EP) e a educação continuada (EC). A EC pode ser entendida como um processo sequencial e acumulativo pelo profissional da saúde das informações técnico-científicas. Pode se dar pela escolarização formal, de experiências laborais, vivências e participação dentro ou fora da esfera institucional. Objetiva, além da aquisição de conhecimentos e habilidades, o aperfeiçoamento profissional e assistencial. A EP, por sua vez, baseia-se em ações de problematização do processo de trabalho em saúde e objetiva a transformação desse processo em suas práticas e organização. Tem como referência as necessidades da população adscrita, a reorganização da gestão 
setorial e a ampliação do vínculo entre formação em saúde e exercício do controle social (Falkenberg et al., 2014).

Ao se considerar a oferta de formação inicial para as EqNasf-AB no início do trabalho, observa-se na Tabela 1, que não houve oferta de formação específica (curso introdutório, oficina de capacitação e reunião formativa) para 50,0\% delas. Das equipes que não receberam formação inicial específica, $58,6 \%$ afirma não ter sido ofertado EP.

As equipes que receberam formação inicial (50,0\%) receberam também maior oferta de EP, sendo esta maior entre as EqNasf-AB que ofertaram formação inicial para todos os profissionais (70,1 \%) (Tabela 1). Esses dados sugerem uma tendência de oferta de EP para as equipes que recebem formação inicial.

\section{Tabela 1}

\begin{tabular}{|c|c|c|c|c|c|}
\hline \multicolumn{3}{|c|}{$\begin{array}{l}\text { Foi oferecida alguma formação específica para o } \\
\text { Nasf quando este iniciou o trabalho? }\end{array}$} & \multicolumn{3}{|c|}{$\begin{array}{l}\text { É oferecida educação permanente para os profissionais do } \\
\text { Nasf? }\end{array}$} \\
\hline Resposta & $\mathrm{N}$ & $\%$ & Resposta & $\mathrm{N}$ & $\%$ \\
\hline \multirow[t]{4}{*}{ Sim, para todos os profissionais } & 41 & 35,3 & Sim, para todos os profissionais & 29 & 70,7 \\
\hline & & & Sim, para alguns profissionais & 03 & 7,3 \\
\hline & & & Não & 09 & 22,0 \\
\hline & & & Total & 41 & 100,0 \\
\hline \multirow[t]{4}{*}{ Sim, para alguns profissionais } & 17 & 14,7 & Sim, para todos os profissionais & 09 & 52,9 \\
\hline & & & Sim, para alguns profissionais & 02 & 11,8 \\
\hline & & & Não & 06 & 35,3 \\
\hline & & & Total & 17 & 100,0 \\
\hline \multirow[t]{3}{*}{ Não } & 58 & 50,0 & Sim, para todos os profissionais & 14 & 24,2 \\
\hline & & & Sim, para alguns profissionais & 10 & 17,2 \\
\hline & & & Não & 34 & 58,6 \\
\hline Total & 116 & 100,0 & Total & 58 & 100,0 \\
\hline
\end{tabular}

Fonte: Os autores

Nota: NASF -36 Núcleo Ampliado de Saúde da Família

É relevante ressaltar que 50\% das EqNasf-AB não receberam nenhuma formação específica no início da atuação na $\mathrm{AB}$, ainda que a literatura explicite que a formação da maioria dos profissionais egressos da academia 
está distante das reais demandas dos usuários do Sistema Único de Saúde, especialmente no âmbito da $\mathrm{AB}$, visto que os conteúdos especializados $\mathrm{e}$ a objetividade tecnológica das ciências da saúde imperam nos currículos acadêmicos (Nunes Junior, Vasconcelos e Arantes, 2017).

No âmbito do tipo de formação inicial fornecida aos profissionais das EqNasf-AB, observou-se que as equipes receberam com maior frequência oficina de capacitação ou reunião informativa $(20,7 \%)$ e um curso 'introdutório' $(20,7 \%)$.

Embora seja relevante conhecer a forma como essas atividades foram realizadas, o instrumento aplicado às equipes não permite elucidar detalhes sobre como se dão os cursos introdutórios, as oficinas de capacitação e reuniões informativas, o que pode ser considerada uma fragilidade do mesmo. Ainda assim, estas atividades parecem se tratar de momentos pontuais, que não propiciam reflexões e discussões com base nas vivências e problemáticas reconhecidas no exercício profissional.

Ainda que seja reconhecida a relevância da formação inicial por suas contribuições para os profissionais do Nasf-AB em exercício, esta é limitada por não propiciar uma reflexão sobre problemas advindos das vivências no contexto profissional. Estar inserido em um ambiente de trabalho no qual lhes seja ofertado EP, potencializa a qualificação destes profissionais à medida que lhes propicia a reflexão de sua prática frente aos problemas tangíveis e complexos inerentes ao seu exercício profissional às EqSFs e à população.

A avaliação da formação específica inicial foi positiva dentre as $58(50,0 \%)$ equipes que receberam essa oferta para todos ou alguns profissionais do NasfAB. Tal avaliação positiva da formação inicial específica ofertada quer boa $(63,8 \%)$ ou muito boa $(27,6 \%)$, pode ser reflexo do reconhecimento da contribuição destas atividades para o cotidiano do trabalho destes profissionais.

Quanto à oferta de EP por municípios, a análise dos dados evidenciou que dos 75 municípios avaliados, a maioria (37) não ofertou EP para as EqNasf$\mathrm{AB}$. Vinte municípios ofertaram EP para todos os profissionais e 18 municípios ofertaram parcialmente EP para os profissionais ou EqNasf-AB. Nesta última categoria estão inclusos municípios em que houve oferta para alguns profissionais de uma mesma equipe ou para algumas equipes de uma mesma cidade (Figura 1). 


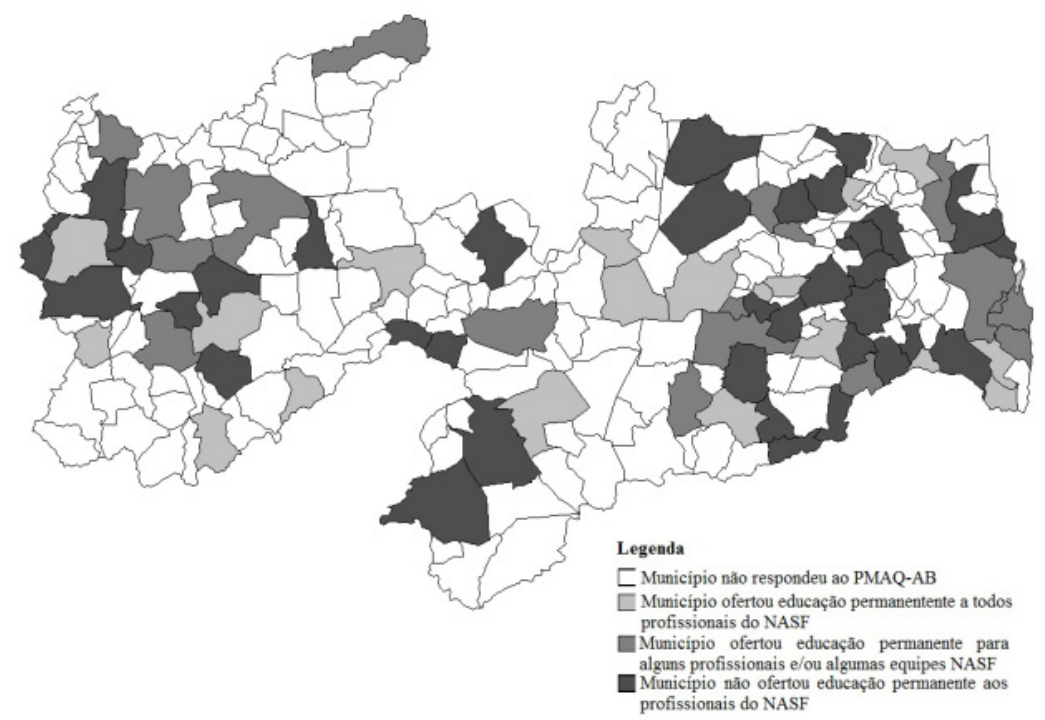

Fonte: Os autores

Nota: PMAQ-AB - Programa Nacional de Melhoria do Acesso e da Qualidade da Atenção Básica; NASF- Núcleo Ampliado de Saúde da Família e Atenção Básica.

Embora a maioria dos municípios avaliados não tenha ofertado EP para os profissionais da equipe Nasf-AB, quando se analisa o quantitativo de equipes, percebe-se que $44,8 \%$ (52) das equipes afirmam ter recebido oferta de educação permanente para todos os profissionais. Em 12,9\% (15) essa oferta foi apenas para alguns profissionais e para 42,2\% (49) a oferta não existiu.

Quando se analisam os municípios de maior porte do estado, percebe-se que em Patos e Cajazeiras houve oferta de EP para todos os profissionais da equipe Nasf-AB, enquanto que em João Pessoa e Campina Grande há equipes em que a oferta foi realizada para todos os profissionais e outras apenas para alguns ou nenhum deles. Esses dados podem sugerir que os municípios de grande porte investem mais em EP quando comparados aos de pequeno porte, o que pode estar relacionado ao maior aporte financeiro dos primeiros.

Também deve ser considerada a limitação do instrumento do PMAQ-AB em trazer dados mais acurados sobre a concepção dos respondentes quanto à concepção de EP, que de acordo com o Ministério da Saúde, consiste em 
uma modalidade que envolve a reflexão sobre o processo de trabalho (Brasil, 2017 b). Ora, se a EP é um processo contínuo, protagonizado pelos profissionais envolvidos, questiona-se: como pode existir numa mesma EqNasf-AB alguns profissionais que recebem essa oferta e outros não, ainda que haja alta rotatividade?

No âmbito dos temas abordados nas atividades de EP ofertadas às 67 EqNasf-AB, observou-se que os mais frequentes foram: organização do processo de trabalho do Nasf $(82,1 \%)$, saúde da criança $(80,6 \%)$, princípios e diretrizes da $\mathrm{AB}(77,6 \%)$, organização do processo de trabalho da $\mathrm{AB}(77,6 \%)$, saúde da mulher $(76,1 \%)$, saúde mental $(74,6 \%)$ e situações de conflitos sociais como: violência, uso de álcool e de outras drogas (73,1\%). Tais temas podem refletir a demanda das EqNasf-AB frente às necessidades dos usuários e das EqSF/EqAB que apoiam, e podem apontar uma fragilidade na formação dos profissionais de saúde. Ainda assim o instrumento não contém informações sobre a forma como os temas são trabalhados com profissionais do Nasf-AB.

Embora o instrumento não esclareça a dinâmica da oferta de EP para os profissionais do Nasf-AB nos municípios paraibanos, observa-se avaliação positiva das equipes que tiveram oportunidade de participar de atividades de EP, o que pode refletir a contribuição desta para a qualificação dos profissionais em exercício na $\mathrm{AB}$.

\section{Planejamento, monitoramento e avaliação das ações}

Quanto ao planejamento e programação das ações, a maioria das EqNasf$A B$ entrevistadas $(89,1 \%)$ referiu realiza-los mensalmente, embora $19,1 \%$ destas tenham negado a existência de documentação comprobatória. Nas demais equipes, $6,4 \%$ os realizam semanalmente, $0,9 \%$ trimestralmente, $0,9 \%$ semestralmente e $2,7 \%$, não os realizam.

Para embasar o planejamento e a avaliação das ações do Nasf-AB, a gestão disponibiliza informações relacionadas aos principais problemas de saúde do território (86,2\%), às principais demandas das EqSF/EqAB $(77,6 \%)$, aos dados epidemiológicos do município (70,7\%), ao perfil da demanda atendida pelo Nasf-AB $(62,9 \%)$, a outras prioridades definidas pela gestão municipal $(55,2 \%)$ e aos desafios apontados na autoavaliação (50,9 \%) (Figura 2). 
Figura 2

Informações disponibilizadas pela gestão municipal para auxiliar as atividades de diagnóstico, avaliação e planejamento das ações.

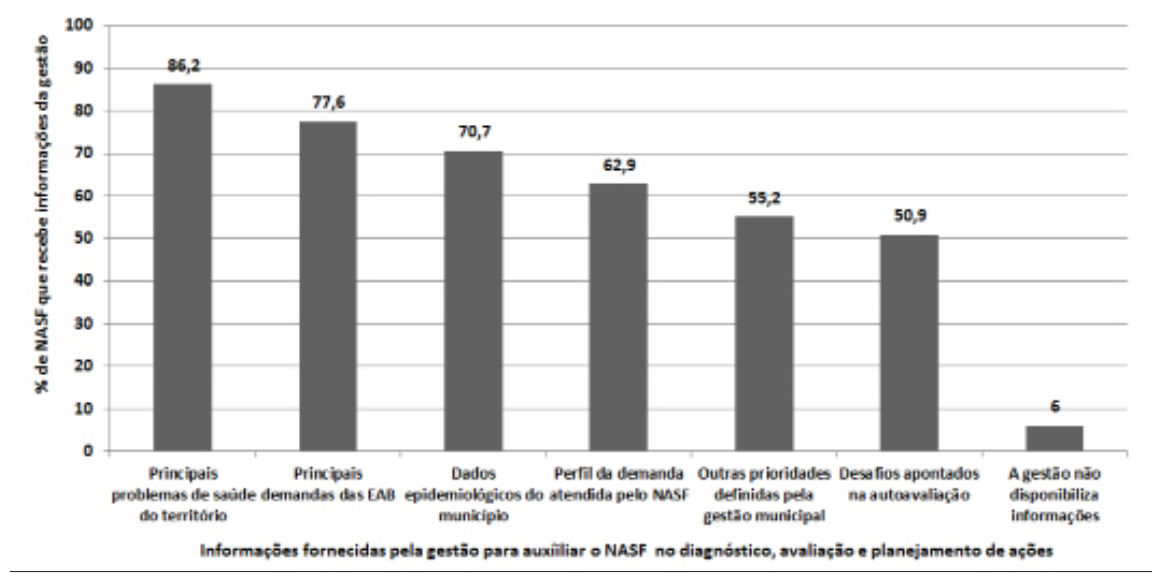

Fonte: Os autores

Nota: $E A B$ - Equipes de Atenção Básica

Independentemente da disponibilização destas informações, a maioria $(83,6 \%)$ das EqNasf-AB entrevistadas afirmou realizar seu planejamento de forma integrada ao planejamento das equipes de $\mathrm{AB}$ que apoiam. Esse dado parece apontar para o trabalho conjunto estre as equipes, o que condiz com os objetivos do Nasf-AB enquanto modalidade de apoio matricial, a saber: ampliação da abrangência das ações e da resolubilidade das EqSF/EqAB (Brasil, 2008; Silva, I. et al., 2017).

Quando analisadas as atividades de monitoramento, percebe-se que 63,3\% dos Nasfs-AB participam do monitoramento e da análise de indicadores e informações de saúde em conjunto com as equipes de $\mathrm{AB}$ apoiadas. Afirmam realizar o monitoramento e análise de indicadores referentes ao seu próprio processo de trabalho $72,7 \%$ das EqNasf-AB, mas apenas $46,5 \%$ possui documento comprobatório.

No âmbito da avaliação do impacto e resultado das ações do Nasf-AB nas equipes apoiadas por meio de indicadores e sinalizadores, as EqNasf-AB declararam avaliar: situações de saúde dos casos compartilhados $(67,2 \%)$, indicadores de saúde da população do território $(62,9 \%)$, número de solicitações de atendimentos desnecessariamente direcionadas ao Nasf (58,6\%), número de encaminhamentos realizados de forma equivocada ou desnecessária para a atenção especializada (50\%) e 12,9\% das equipes declarou não avaliar o impacto/resultado de suas ações nas equipes apoiadas.

Apesar da existência dos indicadores supracitados, apenas 56,4\% das EqNasf-AB afirmou monitorar as solicitações de apoio das equipes de $\mathrm{AB}$ apoiadas, identificando as demandas mais frequentes e o percentual de aten- 
dimento da demanda observada, e somente $32,7 \%$ referiu ter documento que comprove esta monitorização.

Esses dados podem sugerir que há uma compreensão equivocada das EqNasf-AB quanto ao conceito de planejamento, que seria próxima ao senso comum, ou seja, como algo informal e não sistematizado. Essa hipótese é fortalecida à medida que se observa que mais de $80 \%$ das equipes afirmam planejar, mas este percentual diminui nos quesitos monitoramento e avaliação do impacto e resultado de suas ações nas equipes apoiadas. Se o profissional não participa ativamente do planejamento, ele tende a se considerar com pouca responsabilidade pelo resultado final do processo (Bertusso, 2017).

Segundo Matus (1996, apud Paim, 2006), o planejamento é algo sistemático e processual que abrange quatro momentos fundamentais: explicativo, normativo, estratégico e tático-operacional. Tais momentos são dinâmicos e não necessariamente obedecem a uma sequência estática, dada a dinamicidade do cotidiano dos serviços de saúde. Ao momento explicativo caberia identificar e explicar os problemas presentes na situação analisada, bem como, observar as oportunidades para a ação. Nele as perguntas 'quais' (problemas) e 'por $q u \hat{e}^{\prime}$ (ocorrem) deveriam ser respondidas. Ao momento normativo, pertenceria a definição de objetivos, metas, atividades e recursos necessários. Nele há a definição do que deve ser feito. No momento estratégico, seria realizado o balanço entre o que deve ser feito e o que pode ser feito, estabelecendose os cursos de ação para a superação dos obstáculos. E por fim, o momento tático-operacional, caracterizado pelo fazer. Nele a ação se realiza, requer adaptações, flexibilidade, informações, acompanhamento e avaliação (Matus, 1996 apud Paim, 2006).

Para Lacerda, Botelho e Colussi (2012, p. 18), “o exercício sistemático do planejamento potencializa o alcance dos objetivos por reduzir incertezas envolvidas no processo decisório", o que é objetivado no trabalho em saúde. Neste sentido, é necessário reconhecer as fragilidades existentes neste processo e se dispor a executar o planejamento sistemático, em todas as suas etapas, a fim de potencializar e qualificar a assistência e o apoio prestados.

Ainda no âmbito da avaliação das ações, 95,7\% das EqNasf-AB afirmaram ter realizado algum processo de autoavaliação nos últimos 12 meses. Destas, $84,7 \%$ possuem documento que comprove. A maioria das equipes afirma realizar a autoavaliação de maneira formal e sistematizada. O principal instrumento usado no processo de autoavaliação foi a Autoavaliação para melhoria do Acesso e da Qualidade da Atenção Básica (AMAQ) - Nasf (87,9\%). Algumas equipes referiram realizar processo autoavaliativo informal, sem instrumento específico $(8,6 \%)$. A autoavaliação por meio de instrumentos desenvolvidos pela própria equipe Nasf-AB $(7,8 \%)$ e pela Secretaria Municipal de Saúde $(6,0 \%)$ também foram referidas. 
A aplicação do AMAQ - Nasf como principal forma de autoavaliação parece refletir um avanço trazido pelo $P M A Q$ na $A B$, pois sugere que este estimulou processos de acompanhamento e avaliação das equipes de saúde atuantes neste nível de atenção. O PMAQ considera a avaliação uma estratégia para a tomada de decisão e ação imprescindível para melhoria da qualidade das ações de saúde. Neste sentido, a autoavaliação permite o reconhecimento das dimensões positivas e também negativas do trabalho da gestão e das equipes de atenção à saúde, e é um importante instrumento para o planejamento das ações. Com base nela é possível traçar estratégias e iniciativas para mudança e aprimoramento dos serviços (Brasil, 2012a; Gomes, Barbosa e Ferla, 2016).

\section{Integração com a EqSF/EqAB apoiada}

A compreensão da organização do processo de trabalho do Nasf-AB perpassa inevitavelmente pela avaliação da integração destas equipes com as EqSF/EqAB. Neste sentido, serão abordados adiante aspectos relacionados às ações contempladas na agenda dos profissionais do Nasf-AB, às ações desenvolvidas por estes durante os encontros com as EqSF/EqAB, formas de compartilhamento de casos e aspectos relacionados à gestão da demanda e da atenção compartilhada.

No âmbito das ações contempladas na agenda do profissional das EqNasf$\mathrm{AB}$, nota-se uma distribuição relativamente equitativa entre atendimentos domiciliares $(99,1 \%)$, atividades de educação em saúde $(98,3 \%)$ e consultas individuais dos profissionais do Nasf-AB (96,6\%). Além destas, os profissionais realizam consultas compartilhadas com a EqSF/EqAB e com profissionais da própria EqNasf-AB $(91,4 \%)$, atividades comunitárias $(90,5 \%)$, discussões de casos $(90,5 \%)$, grupos terapêuticos $(83,6 \%)$ e um menor percentual $(79,3 \%)$ realizam ações de educação permanente (Figura 3).

\section{Figura 3}

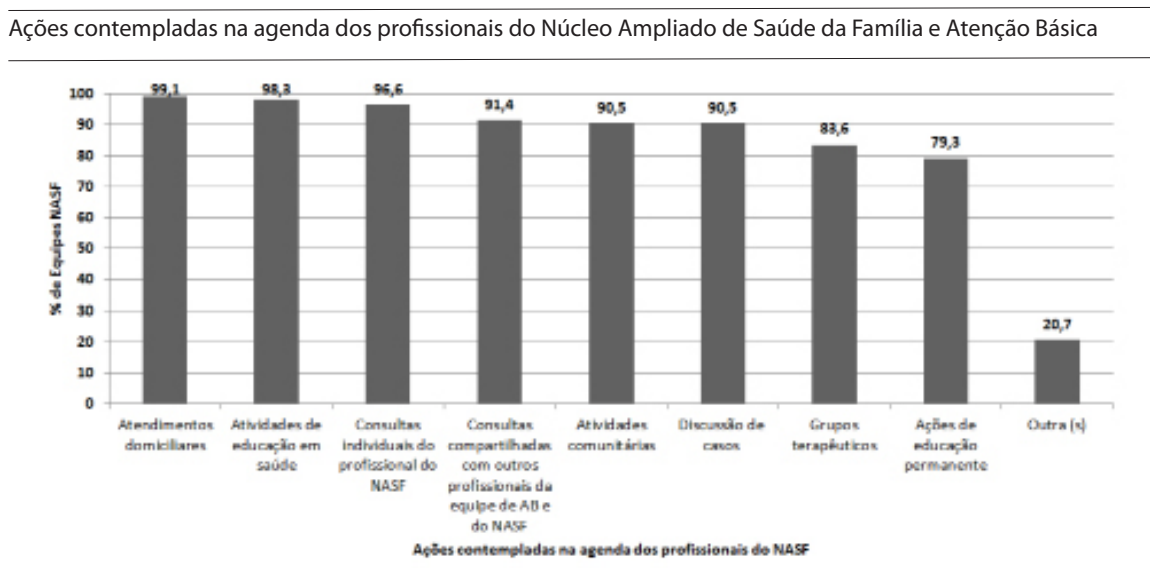


Embora a agenda do Nasf-AB pareça contemplar atividades integradas com as EqSF/EqAB, nota-se que o instrumento não esclarece se os atendimentos domiciliares são realizados pelo Núcleo de forma individual ou integrada. Além disso, apesar de muito próximos, o percentual de consultas individuais dos profissionais do Núcleo ultrapassa o de consultas compartilhadas com as equipes de referência e as discussões de casos. Esse dado pode refletir que o Nasf-AB atua como um agente de ampliação da equipe de $A B$, não só no âmbito do apoio matricial, mas abrindo espaço para atendimento de uma demanda antes reprimida pelo quantitativo reduzido de profissionais nas equipes, diante do excesso e complexidade da demanda de trabalho (Sundfeld, 2010, Silva, I., 2017).

Nos encontros entre as EqNasf-AB e as EqSF/EqAB as atividades desenvolvidas são: visitas domiciliares $(95,7 \%)$, discussão de casos passíveis de encaminhamento aos outros pontos de atenção $(94,8 \%)$, articulação com as equipes de referência, de ações intersetoriais com outros pontos da rede $(93,1 \%)$, organização de intervenções no território em conjunto com as equipes apoiadas $(90,5 \%)$, discussão de casos, eventos sentinelas, casos difíceis e desafiadores $(87,9 \%)$, consultas individuais do Núcleo $(87,1 \%)$, discussões sobre o processo de trabalho das equipes e do apoio ofertado pelas EqNasf-AB (87,1\%), definição de critérios de acesso, fluxos e atribuições de cada profissional $(84,5 \%)$, consultas compartilhadas em conjunto com as $\mathrm{EqSF} / \mathrm{EqAB}(82,7 \%)$, atividades de educação permanente em conjunto aos profissionais das equipes apoiadas $(73,3 \%)$, monitoramento e avaliação dos resultados da atenção compartilhada $(62,9 \%)$ e construção conjunta de projetos terapêuticos $(56,9 \%)$ (Tabela 2$)$.

Diante deste dado destacam-se: a inserção, no instrumento, de consultas individuais da EqNasf- $\mathrm{AB}$ nas atividades desenvolvidas nos encontros entre as EqNasf-AB e as EqSF/EqAB e o baixo percentual de construção conjunta de projetos terapêuticos. Estes dados fortalecem a hipótese da fragilidade na integração entre as EqNasf-AB e EqSF/EqAB na assistência.

Tabela 2

\begin{tabular}{lcc}
\hline Relação das atividades desenvolvidas pelas EqNasf-AB nos encontros com as EqSF/EqAB & \\
\hline Atividades desenvolvidas nos encontros das EqNasf e EqSF/EqAB & $\mathrm{N}$ \\
\hline Realizam visitas com os profissionais da sua equipe & 111 & 95,7 \\
Discussão de casos que poderão gerar encaminhamentos a outros pontos de atenção & 110 & 94,8 \\
Articulação com as equipes AB, de ações com outros pontos da rede de saúde e intersetorial & 108 & 93,1 \\
Organizam intervenções no território em conjunto com sua equipe & 105 & 90,5 \\
Discussão de casos, eventos sentinelas, casos difíceis e desafiadores & 102 & 87,9 \\
Consultas individuais do profissional do Nasf-AB & 101 & 87,1 \\
Discussões sobre o processo de trabalho da EqSF/EqAB e também do próprio apoio do Nasf-AB à equipe & 101 & 87,1 \\
Definição de critérios de acesso, fluxos, atribuições de cada profissional & 98 & 84,5
\end{tabular}




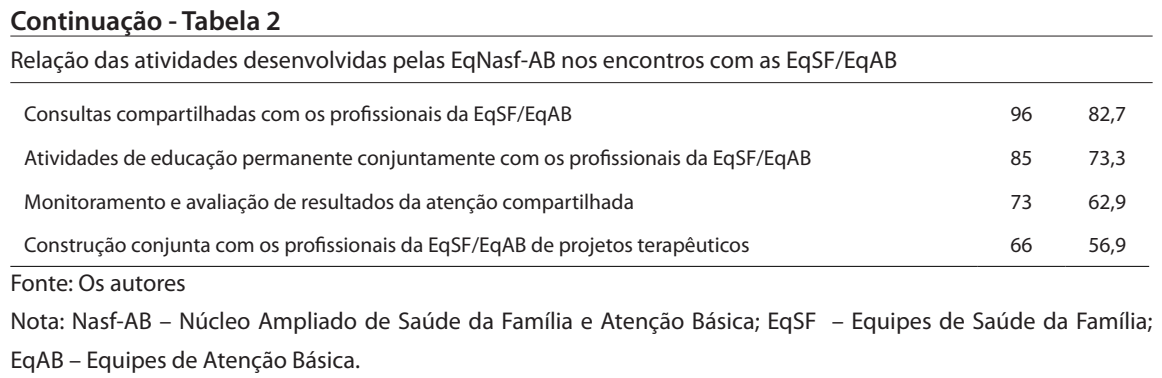

A construção do projeto terapêutico singular consiste em "um recurso clínico e gerencial importantíssimo, cuja construção é privilegiada para o apoio matricial e, portanto, para o trabalho dos profissionais do Nasf"' (Brasil, 2010, p. 27). Este recurso, entretanto, requer que as equipes realizem discussões de casos clínicos para sua construção.

Quando se analisam as formas de compartilhamento de casos em situações de necessidade de apoio, observa-se que estes se dão por meio de discussão de casos $(37,1 \%)$, encaminhamentos por escrito $(34,5 \%)$, consultas compartilhadas $(33,6 \%)$, contato telefônico $(32,8 \%)$ e agendamento de consultas diretamente na agenda do profissional do Nasf-AB (30,2\%) (Figura 4).

Um dado relevante que muito contribui com essa análise provém da análise do módulo II do $2^{\circ}$ ciclo de avaliação externa do PMAQ-AB, aplicado às EqSF/EqAB. Neste módulo, há questões referentes à avaliação destas equipes sobre o apoio recebido do Nasf-AB. Há a mesma questão sobre a forma de compartilhamento de casos entre as equipes. No entanto, a resposta obtida das $\mathrm{EqSF} / \mathrm{EqAB}$ foi que a maioria dos compartilhamentos $(71,3 \%)$ se dava por intermédio de encaminhamentos por escrito.

$\mathrm{O}$ desencontro de informações das EqNasf-AB e EqSF/EqAB também reflete a fragilidade na integração entre elas. Além disso, encaminhamentos por escrito e consultas diretamente na agenda do profissional do Nasf-AB como opções de compartilhamento de casos fortalecem a provável debilidade no exercício de discussões de caso, o que pode contribuir para o baixo percentual de construção de projetos terapêuticos para os indivíduos e famílias assistidas. 


\section{Figura 4}

Distribuição das formas de compartilhamento de casos com as equipes de saúde da família/equipes de atenção básica quando há necessidade de apoio

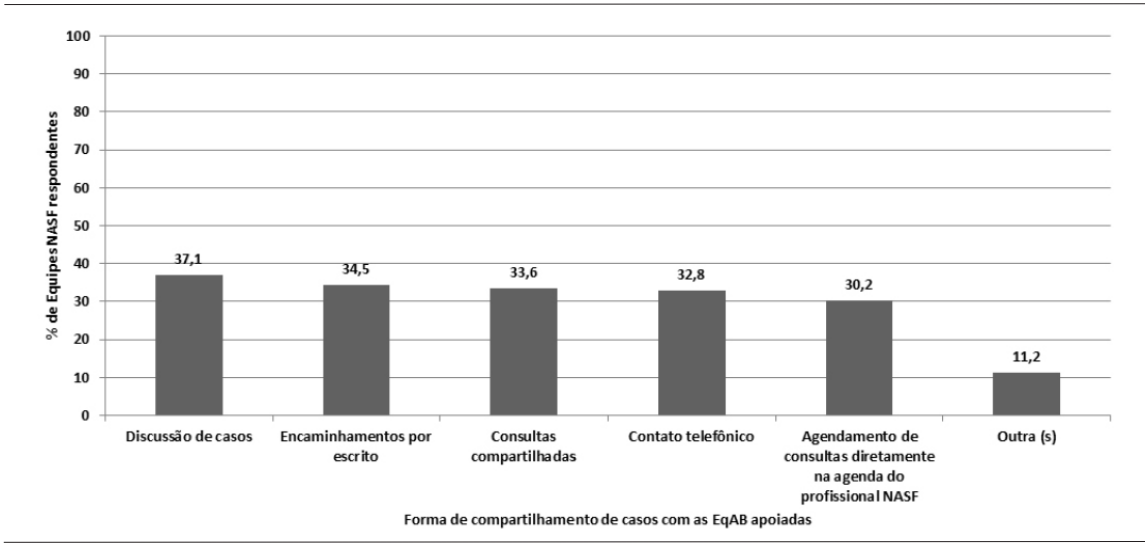

Fonte: Os autores

No âmbito da demanda e da gestão compartilhada, foi avaliado se o Nasf$\mathrm{AB}$ monitorava as solicitações de apoio das equipes apoiadas, identificando as demandas mais frequentes e o percentual de atendimento da demanda observada. A maioria das equipes afirmou realizá-lo, 56\% das equipes o fazia sempre e $22,4 \%$ apenas algumas vezes. No entanto, apenas $32,8 \%$ das equipes afirmou possuir documento que comprove a realização do monitoramento.

Além de indicar uma fragilidade no registro, este dado reflete a debilidade na capacidade de gestão do trabalho do Nasf-AB.

\section{Conclusão}

A análise da organização do processo de trabalho das EqNasf-AB, possibilitou o diagnóstico de algumas limitações relacionadas à oferta de EP, à ausência de planejamento sistematizado e a uma possível desarticulação com as equipes apoiadas.

O déficit na oferta de EP para as EqNasf-AB ocorre principalmente nos municípios de menor porte. Tal condição aponta para a necessidade de investimento da gestão nesta modalidade de educação em saúde, a fim de potencializar o apoio matricial ofertado por estas equipes e, consequentemente, melhorar a qualidade da assistência na $\mathrm{AB}$. 
Quanto ao planejamento das ações, a aparente inexistência de sistematização do processo, evidenciado pela fragilidade nas etapas de monitoramento e avaliação, expõe a urgente necessidade de fortalecimento do planejamento estratégico, como ferramenta para potencializar a resolutividade das ações das equipes frente à demanda da população assistida.

Adicionalmente, a fragilidade na integração entre as EqNasf-AB e as EqSF/ $\mathrm{EqAB}$, evidenciada pela predominância de ações individuais das EqNasf-AB, em detrimento das ações conjuntas, bem como pelas formas de compartilhamento de casos (encaminhamentos por escrito e agendamento de consultas diretamente na agenda dos profissionais do Nasf-AB), aponta para necessidade de investimento nas discussões de caso e na construção conjunta de projetos terapêuticos singulares, a fim de fortalecer a integralidade da assistência. Além disso, mostra a necessidade de fortalecimento da atuação das EqNasf-AB no âmbito da gestão do trabalho.

$\mathrm{O}$ conhecimento das fragilidades inerentes à organização do processo de trabalho do Nasf-AB, abordadas neste estudo, podem subsidiar proposições de estratégias para potencialização do apoio matricial ofertado por estas equipes. Ainda assim, sugere-se o desenvolvimento de estudos qualitativos que possibilitem conhecer os impedimentos à execução do planejamento estratégico e à oferta de EP no cotidiano destas equipes.

\section{Colaboradores}

Todas as autoras contribuíram igualmente com produção da pesquisa; produção e análise dos dados; e elaboração e revisão final do artigo.

\section{Financiamento}

O estudo foi desenvolvido no âmbito do Programa de Bolsas de Iniciação Científica na Universidade Federal da Paraíba, financiado pelo Conselho Nacional de Desenvolvimento Científico e Tecnológico (CNPq). 


\section{EL PROCESO DE TRABAJO DEL NÚCLEO AMPLIADO DE SALUD DE LA FAMILIA Y ATENCIÓN BÁSICA}

Resumen El crecimiento de la implantación del Núcleo Ampliado de Salud de la Familia y Atención Básica en Paraíba y su influencia en la mejora de la calidad de la asistencia de los equipos de atención primaria, hace relevante conocer la organización del proceso de trabajo de dichos núcleos. El estudio analizó acciones de planificación, oferta de formación inicial y de educación permanente e integración con los equipos que se apoya. Metodológicamente es descriptivo, cuantitativo, de corte transversal. Los datos se obtuvieron a partir del módulo IV del $2^{\circ}$ ciclo de evaluación externa del Programa de Mejora y Acceso de Calidad de la Atención Básica. El mapa coroplético se construyó en TabWin y el análisis descriptivo se realizó en el IBM SPSS Statistics 20.0. Respecto a la oferta de formación inicial y de educación permanente para el Núcleo, se observó que los equipos que recibieron la primera (50\%) tuvieron una mayor frecuencia de oferta de la segunda $(74,1 \%)$, y se ubicaban en municipios de mayor tamaño. La formación inicial tuvo como actividades más frecuentes los talleres de capacitación/reunión informativa (20,7\%) y curso introductorio $(20,7 \%)$. Se observa una fragilidad en los registros y en la ejecución de la planificación sistematizada, principalmente en la monitorización y evaluación de las acciones. La integración entre los equipos Núcleo de Apoyo a la Salud de la Familia y los equipos de referencia es frágil y escapa de la presunción conceptual de apoyo matricial.

Palavras clave atención básica; salud de la familia; planificación en salud; educación permanente.

\section{Referências}

BERTUSSO, Francielle R. A contribuição do programa nacional de melhoria do acesso e da qualidade da atenção básica (PMAQ-AB), no processo de trabalho das equipes de saúde da família. 2017. 112f. Dissertação (Mestrado em Biociências da Saúde)-Universidade Estadual do Oeste do Paraná, Cascavel, 2017.

BRASIL. Ministério da Saúde. Autoavaliação para melhoria do acesso e da qualidade da atenção básica: AMAQ. Brasília: Ministério da Saúde, 2012a. 134p. Disponível em: <http://189.28.128.100/dab/docs/geral/amaq. pdf>. Acesso em: 9 ago. 2017.

BRASIL. Ministério da Saúde. Portaria n. 154, de 24 de janeiro de 2008. Cria os Núcleos de Apoio à Saúde da Família. Diário Oficial da União, Brasília, DF, 4 mar. 2008. Seção I, n.43, p. 38.40. Republicada por ter saído com incorreção no DOU n.18, de 25 de janeiro de 2008, p. 47.49. Disponível em: <\&lt;http:// bvsms.saude.gov.br/bvs/saudelegis/gm/2008/ prt0154_24_01_2008.html\&gt; > Acesso em: 24 ago. 2015.
BRASIL. Ministério da Saúde. Portaria n. 1.654 de 19 de julho 2011. Institui no âmbito do Sistema Único de Saúde, o Programa Nacional de Melhoria do Acesso e da Qualidade da Atenção Básica (PMAQ-AB) e o Incentivo Financeiro do PMAQ-AB, denominado Componente de Qualidade do Piso de Atenção Básica Variável - PAB Variável. Diário Oficial da União, Brasília, DF, 2011. Disponível em: $<$ http://bvsms.saude.gov.br/bvs/saudelegis/ gm/2011/prt1654_19_07_2011.html>. Acesso em: 1 nov. 2015.

BRASIL. Ministério da Saúde. Portaria n. 3.124, de 28 de dezembro de 2012. Redefine os parâmetros de vinculação dos Núcleos de Apoio à Saúde da Família (NASF) Modalidades 1 e 2 às Equipes Saúde da Família e/ou Equipes de Atenção Básica para populações específicas, cria a Modalidade NASF 3, e dá outras providências. Diário Oficial da União, Brasília, DF, 31 dez. 2012b. Seção 1, n. 251, p. 223. Disponível em: <http://bvsms.saude.gov.br/ bvs/saudelegis/gm/2012/prt3124_28_12_2012. html>. Acesso em: 15 set. 2015. 
BRASIL. Ministério da Saúde. Portaria nº 2.436, de 21 de setembro de 2017a. Aprova a Política Nacional de Atenção Básica, estabelecendo a revisão de diretrizes para a organização da Atenção Básica, no âmbito do Sistema Único de Saúde (SUS). Diário Oficial da União, Brasília, DF, 2011. Disponível em: <http://www.foa. unesp.br/home/pos/ppgops/portaria-n-2436. pdf $>$. Acesso em: 5 fev. 2018.

BRASIL. Ministério da Saúde. Secretaria de Atenção à Saúde. Departamento de AtençãoBásica. Política Nacional de Atenção Básica. Brasília: Ministério da Saúde, 2017b. 38 p.

BRASIL. Ministério da Saúde. Secretaria de Atenção à Saúde. Departamento de AtençãoBásica. Departamento de Ações Programáticas Estratégicas. Saúde mental. Brasília: Ministério da Saúde, 2013. 176 p. (Cadernos de Atenção Básica; n. 34).

BRASIL. Ministério da Saúde. Secretaria de Atenção à Saúde. Departamento de AtençãoBásica. Diretrizes do NASF. Brasília: Ministério da Saúde, 2010. 152 p. (Cadernos de Atenção Básica; 27).

BRASIL. Ministério da Saúde. Secretaria de Atenção à Saúde. Departamento de Atenção Básica. Núcleo de apoio à saúde da família. Brasília: Ministério da Saúde, 2014. Volume 1. (Cadernos de Atenção Básica; n. 39).

CAMPOS Gastão W. S., DOMITTI, Ana C. Apoio matricial e equipe de referência: uma metodologia para gestão do trabalho interdisciplinar em saúde. Cadernos de Saúde Pública, Rio de Janeiro, v. 23, n. 2, p. 399-407, 2007.

CHIMARA, Marília B. et al. Gestão do sistema de saúde do município de São Paulo com base nos parâmetros de avaliação do PMAQ-AB: estudo de caso na microrregião de cidade Tiradentes. Revista de Gestão em Sistemas de Saúde, São Paulo, v. 2, n. 2, p. 174-97, jul./dez. 2013.

FALKENBERG, Mirian B. et al. Educação em saúde e educação na saúde: conceitos e implicações para a saúde coletiva. Ciência \&
Saúde Coletiva, Rio de Janeiro, v. 19, n. 3, p. 847-852, mar. 2014. Disponível em: <http:// www.scielosp.org/scielo.php?script $=$ sci arttext\&pid=S1413-81232014000300847\&lng $=\mathrm{en} \& \mathrm{nrm}=\mathrm{iso}>$. Acesso em: 22 jun. 2017.

FRANÇA, Bruna D. et al. Núcleo de apoio à saúde da família: um dispositivo para a prática de educação permanente. In: REUNIÃO ANUAL DA SBPC, 69, 2017, Belo Horizonte. Anais... Belo Horizonte: UFMG, 2017.

GOMES, Luciano B.; BARBOSA, Mirceli G.; FERLA, Alcindo A. Atenção Básica: olhares a partir do Programa Nacional de Melhoria do Acesso e da Qualidade (PMAQ-AB). Porto Alegre: Rede UNIDA, 2016. 357 p.

LACERDA, Josimari T.; BOTELHO, Lúcio J.; COLUSSI, Cláudia F. Planejamento na Atenção Básica. Curso de Especialização Multiprofissional em Saúde da Família. Florianópolis: Universidade Federal de Santa Catarina, 2012.

MARTINIANO, Claudia S. et al. Avaliação do processo de implantação das equipes dos Núcleos de Apoio à Saúde da Família. Journal of Nursing UFPE on line, Recife, v. 7, n. 1, p. 53-61, Jan. 2013.

NUNES JÚNIOR, Josemar R.; VASCONCELOS, Cinthia R.; ARANTES, Ana C. P. Formação para o trabalho no SUS: um olhar para o Núcleo de Apoio à Saúde da Família e suas categorias profissionais. Cadernos de Educação, Saúde e Fisioterapias, s.l., v. 4, n. 7, Jan. 2017.

ONOCKO-CAMPOS, Rosana T. et al. Avaliação da qualidade do acesso na atenção primária de uma grande cidade brasileira na perspectiva dos usuários. Saúde em Debate, Rio de Janeiro, v. 38, n. esp., p. 252-264, Out. 2014. Disponível em: <http://www.scielo.br/scielo. php?script=sci_arttext\&pid $=$ S01031104201 $4000600252 \& \operatorname{lng}=\mathrm{en} \& \mathrm{nrm}=\mathrm{iso}>$. Acesso em: 27 abr. 2018.

PAIM, Jairnilson S. Planejamento em saúde para não especialistas. In: CAMPOS, Gastão W. Tratado de Saúde Coletiva. Rio de Janeiro: HUCITEC/Ed. Fiocruz, 2006. p. 767-782. 
PINTO, Hêider A.; SOUSA, Allan N. A.; FERLA, Alcindo A. O Programa Nacional de Melhoria do Acesso e da Qualidade da Atenção Básica: várias faces de uma política inovadora. Ministério da Saúde. Saúde em Debate, Rio de Janeiro, v. 38, p. 358-372, 2014. Número Especial.

SILVA, Isabelle C. B. et al. Processo de trabalho entre a Equipe de Atenção Básica e o Núcleo de Apoio à Saúde da Família. Revista Brasileira de Medicina de Família e Comuni- dade (RBMFC), Rio de Janeiro, v. 12, n. 39, p. 1-10, Jan. 2017.

SILVA, Laís A. B. et al. Analysis of the distribution and composition of family health support centres in the state of Paraíba, Brazil. International Journal of Development Research, s.l., v. 7, n. 12, p. 17416-17424, dez. 2017.

SUNDFELD, Ana C. Clínica ampliada na Atenção Básica e processos de subjetivação: relato de uma experiência. Physis: Revista de Saúde Coletiva, Rio de Janeiro, v. 20, n. 4, p. 1079-1097, 2010. 